\title{
Identidad, reconocimiento y participación. Ordenamiento territorial y justicia ambiental en las zonas rurales de Medellín (Colombia)*
}

\author{
Recibido: 2015-03-26 -Enviado a pares: 2015-04-08 \\ Aprobado por pares: 2015-04-27 Aceptado: 2015-05-08 \\ Carlos Javier Egio Rubio** \\ Eryka Yuvelyre Torrejón Cardona*** \\ María Camila Muñoz Arias*** \\ Loraine Cumplido Rodríguez ${ }^{* * * *}$
}

\section{Resumen}

Este artículo presenta los resultados de una investigación donde se usan la justicia ambiental y la comunicación para el cambio social como ejes teóricos. Se desarrolla con metodologías participativas en el contexto de las zonas rurales de Medellín durante el 2013. Su objetivo es identificar y clasificar las percepciones de los actores sociales que participan en la política de ordenamiento territorial. Los resultados que se encuentran son: (i) una gran diversidad de actores sociales, (ii) un alto nivel de dispersión de acciones en todo el territorio rural y (iii) unas identidades locales en constante cambio y reconstrucción, pero siempre asociadas a la ruralidad.

El artículo finaliza subrayando algunas percepciones colectivas en respuesta a los efectos ambientales del modelo de expansión urbana y cómo los actores sociales de las cinco

Este artículo es producto del proyecto "Los corregimientos de Medellín frente a las externalidades urbanas", que ha sido financiado con recursos del Comité para el Desarrollo de la Investigación (CODI) en la Convocatoria de Ciencias Sociales, Humanidades y Artes (versión 2012) y del proyecto CODI E01756, 2013-2014 para la Sostenibilidad del Grupo de investigación Medio Ambiente y Sociedad (MASO) de la Universidad de Antioquia. Asimismo, hace parte del convenio Universidad Complutense de Madrid-Universidad de Antioquia para pasantía de investigación doctoral, con apoyo del Observatorio Internacional de Ciudadanía y Medio Ambiente Sostenible (CIMAS). Está adscrito al Grupo Medio Ambiente y Sociedad. (COLOMBIA)

** Pregrado en Ciencias Ambientales y Periodismo en la Universidad de Murcia (España), magíster en Comunicación, Cambio Social y Desarrollo por la Universidad Complutense de Madrid y estudiante de doctorado en Sociología en esta última universidad. Investigador principal en el proyecto "Los corregimientos de Medellín frente a las externalidades urbanas", Universidad de Antioquia. Correo electrónico: cjegio@gmail.com

.** Pregrado en Sociología en la Universidad de Antioquia (Colombia), Doctora en Geografía en la Universidad Autónoma de Barcelona. Docente de Sociología e investigadora del Grupo Medio Ambiente y Sociedad de la Universidad de Antioquia. Co investigadora y asesora local del proyecto "Los corregimientos de Medellín frente a las externalidades urbanas". Correo electrónico: eryka.torrejon@siu.udea.edu.co

...*. Estudiante de último semestre de Periodismo de la Universidad de Antioquia, apoyo como estudiante en formación del proyecto "Los corregimientos de Medellín frente a las externalidades urbanas".

***** Comunicadora de la universidad de Antioquia, integrante del grupo Medio Ambiente y Sociedad, apoyo como estudiante en formación del proyecto "Los corregimientos de Medellín frente a las externalidades urbanas". 
zonas rurales del municipio de Medellín cuentan con un legado de procesos de participación comunitaria con potencialidad para incidir con acciones locales en una justicia ambiental. Palabras clave: Medellín, justicia ambiental, zonas rurales, comunicación para el cambio social, participación.

\title{
Local identities, recognition and participation. Planning and management of rural areas of Medellin (Colombia).
}

\begin{abstract}
This article presents the results of an investigation where environmental justice and communication for social change are used for social change as theoretical concepts. It develops with participatory methodologies in the context of rural areas of Medellin in 2013. Their goal is to identify and classify the perceptions of the social actors involved in the policies. The results found are (i) a wide range of social actors, (ii) a high level of dispersion of actions in all rural territories and (iii) a few local identities in constant change and reconstruction, but always associated with rural life.

The article concludes by highlighting some collective perceptions in response to environmental effects of the model of urban expansion and how social actors in five rural areas of the municipality of Medellín have a legacy of community participation processes with the potentiality to influence local actions in a environmental justice.
\end{abstract}

Key words: Medellin, Environmental Justice, rural zones, Communication for Social Change, Participation.

\section{Identidade, reconhecimento e participação. Ordenamento territorial e justiça ambiental na área rural de Medelin (Colômbia).}

\section{Resumo}

Este artigo apresenta os resultados de uma pesquisa onde se usa a justiça ambientale a comunicação para a mudança social como eixos teóricos. Desenvolve-se com metodologias participativas no contexto das zonas rurais de Medellín durante 2013. Seu objetivo é identificar e classificar as percepções dos atores sociais que participam da politica de ordenamento territorial. Os resultados que se encontram são (i) uma diversidade de atores sociais, (ii) um alto nível de dispersão de açõesem todo o território rural e (iii) umas identidades locais em constante mudança e reconstrução.

O artigo finaliza salientando algumas percepções coletivas em resposta aos efeitos ambientais do modelo de expansão urbana e como os atores sociais das cinco zonas rurais do município de Medellín contam com um legado de processos de participação comunitária com potencialidades para incidir com ações locais em uma justiça ambiental.

Palavras chaves: Medellín, Justiça Ambiental, Zonas Rurais, comunicação para a mudança social, participação. 


\section{Introducción}

En este artículo se presentan los resultados de una investigación que partía fundamentalmente de una pregunta: ¿Cómo perciben los actores sociales de las zonas rurales del municipio de Medellín el fenómeno de expansión de la ciudad? Pregunta que se ha pretendido resolver mediante el uso de metodologías de investigación participativa y de la comunicación para el cambio social. Así, el objetivo de investigación es identificar las percepciones y discursos relacionados con la expansión de la ciudad de Medellín y sus efectos sociales, culturales, ambientales y económicos en sus cinco corregimientos.

El artículo se compone de cuatro partes: en la primera, se hace una breve contextualización del objeto de estudio y se presentan las principales etapas que han dado lugar a una política pública de ordenamiento territorial rural; en la segunda, se incluyen las nociones teóricas centrales del artículo (justicia ambiental-justicia espacial, espacio rural y comunicación para el cambio social), a la vez que se indican de forma sucinta la metodología y las técnicas de investigación empleadas; la tercera parte está destinada a la exposición de los principales resultados, y la cuarta, y última parte, a las conclusiones.

\subsection{Contexto, un territorio rural metropolitano}

Para comprender mejor las percepciones surgidas en las entrevistas, que suponen el cuerpo principal de análisis de este artículo, hay que describir algunas características del municipio de Medellín, como que este alberga el núcleo de población más importante del Área Metropolitana del Valle de Aburrá, un área urbana que ocupa la mayor parte de una depresión de la Cordillera Central de los Andes cercada por montañas a este y oeste. A pesar de que el valle, debido a esta orografía, cuenta con poco espacio disponible para un crecimiento ordenado y sin riesgo, acoge al $56 \%$ de la población del departamento de Antioquia (Piedrahíta y González, 2010, p. 221).

Aunque nos centraremos exclusivamente en este municipio, para empezar a definir la relación entre el territorio urbano del valle y el rural, y puesto que vamos a trabajar con la noción de justicia ambiental, no está de más indicar que la huella ecológica del área metropolitana es 66,6 veces mayor que su superficie ocupada directamente. Es decir, que para cubrir su dependencia ecológica los habitantes de este territorio necesitan utilizar recursos naturales de una ecorregión que supera incluso los límites del departamento (Agudelo, 2002, p. 11).

En 2005, el municipio de Medellín contaba con una población total de 2.219.861 personas, 2.175 .681 de las cuales vivían en la zona urbana en $106 \mathrm{~km}^{2}$ (aproximadamente para la cabecera) y tan solo 38.813 personas en la zona rural ${ }^{2}$ en $270 \mathrm{~km}^{2}$ (DANE, 2005, p. 473). Es decir, aunque la población rural sea casi cincuenta veces menor que la población urbana, el área rural representa en términos un territorio extenso, básico para el equilibrio ambiental del municipio y culturalmente significativo, más, si tenemos en cuenta que se trata un territorio en el que, tras décadas caracterizadas por una prolongada violencia interna, se están haciendo importantes esfuerzos desde la administración para elevar las cotas de inclusión social. 
Esta distribución obedece, sin embargo, a un fenómeno muy reciente en términos históricos pues los datos poblacionales muestran un crecimiento acelerado en la primera mitad del siglo XX y un crecimiento permanente durante la segunda (Departamento de Geología Universidad EAFIT, 2010, p. 69). En cuanto a los cinco corregimientos, aunque con características muy dispares entre sí, que van desde la casi plena conurbación y el predominio de prácticas urbanas hasta la economía agrícola y la separación por varios kilómetros de la ciudad, estos constituyen una zona de borde o encuentro entre la ruralidad y sus formas de vida tradicionales y las formas de vida más urbanas. Se trata de un espacio heterogéneo en el que pueden encontrarse actividades y usos como los industriales, agrícolas, recreativos, pecuarios, residenciales o comerciales.

De los 376,21 km² del municipio de Medellín, 102,10 km² se consideran como suelo urbano, 4,01 km² como áreas de expansión urbana y 270,10 km² como suelo rural (Departamento Administrativo de Planeación, 2006, p. 84). De hecho, en la última década, la zona rural ha ido adquiriendo mayor relevancia en las políticas municipales, quizá respondiendo a un aumento de la presión sobre esta relacionado con una mayor demanda de suelo para fines residenciales, industriales y de servicios, que ha ocasionando que la renta del suelo haya ido en aumento.

A esto hay que sumar la necesidad de disponer de corredores de transporte para facilitar la comunicación de Medellín con otras regiones tanto de Colombia como de Latinoamérica y del resto del mundo (Departamento Administrativo de Planeación, 2012, p. 417). Esto último es lo que sucede con los túneles de Oriente (en proyecto) y Occidente, ambos cuestionados por algunos colectivos sociales por un posible impacto sobre los acuíferos y otros recursos naturales asociados como son la flora, la fauna, los bosques y los subproductos de estos. Esta tendencia se agudiza en gran medida con el aumento de la huella ecológica a la que aluden los expertos, la cual requiere de medidas de regulación y de concertación en el ordenamiento y la planificación del territorio del valle interandino donde se hospedan los habitantes de Medellín.

La población de los corregimientos no ha sido ajena a este fenómeno. Por la proximidad geográfica al entorno urbano de Medellín, el territorio de estudio puede describirse como rural metropolitano. Esa cercanía ha generado importantes transformaciones sociales y culturales, producto de la convivencia entre la población rural tradicional, la población rural de nueva generación y la citadina que de forma creciente ha ocupado y habita estos espacios próximos al centro metropolitano³ (Agudelo, 2012, p. 561).

Es decir, los habitantes tradicionales de los corregimientos, ante la expansión urbana, se han visto enfrentados a la imposibilidad de cubrir las demandas provenientes de la presión ejercida sobre las zonas rurales dando como resultado "fuertes transformaciones territoriales y produciendo paisajes en transición de rurales a urbanos en donde se yuxtaponen usos y actividades agrícolas, residenciales, industriales y de ocio" (Cano, 2012, p. 15). 


\subsection{Evolución de las políticas de ordenamiento territorial}

Puesto que, tal y como se explicará en el marco teórico, dos de los elementos clave de las nociones de justicia ambiental y espacial son el reconocimiento y la capacidad de participación y decisión real, es imprescindible aportar un par de pinceladas sobre la evolución que han tenido en el período comprendido entre 2004 y 2013 las políticas de ordenamiento territorial del municipio. No obstante, por razones de extensión, se indicarán solamente algunos hechos distintivos entre la primera y la segunda generación de planes de ordenamiento territorial en la ciudad.

Hay que destacar que en el período indicado se ha pasado de un Plan de Ordenamiento Territorial (a partir de ahora POT) (1999) centrado en la zona urbana a una versión mejorada, adaptada y vinculada a las zonas rurales (2006). De hecho, en el momento en que se redactaba este artículo se elaboraba una nueva versión del POT (2013). Así, la revisión de 2006 propició una política rural participativa que se reflejó, entre otras cosas, en la generación y puesta en marcha de la Gerencia de Corregimientos, cuyas funciones fueron trasladadas al Departamento Administrativo de Planeación Municipal en el 2011. Esta serie de cambios han ido acompañados de una permanente y dispersa participación de los pobladores rurales sobre los efectos que conlleva la clasificación del suelo de su territorio.

Entre los primeros instrumentos de la política rural participativa, destaca el Plan de Estrategias Corregimentales -en adelante Plan ECO- que en 2003 encargó el Departamento Administrativo de Planeación a la escuela del Hábitat de la Universidad Nacional de Colombia para la elaboración de diagnósticos y la propuesta de alternativas para el manejo sostenible del territorio en cada uno de los corregimientos. Se trataba de un esfuerzo importante para alimentar el Plan de Ordenamiento Territorial, mejorando la comprensión y gestión de los espacios menos urbanos del municipio.

Más adelante, a partir del Decreto Nacional 3600 de 2007, aparecía en escena una nueva figura que complementaría exclusivamente el componente rural del POT. Los llamados Planes Especiales de Ordenamiento Corregimental (PEOC) perseguirían reconocer el nuevo protagonismo de las áreas menos urbanas con el fin de paliar algunos fenómenos negativos identificados en el Plan ECO. Elaborados participativamente entre 2009 y 2010 (Pérez, 2011, p. 40), los PEOC fueron aprobados y adoptados por parte del Consejo Municipal. Sin embargo, estos eran solo una orientación para futuras modificaciones del Plan de Ordenamiento Territorial y carecian de un carácter vinculante.

En resumen, en el período de estudio se encuentran tres etapas significativas para el ordenamiento del territorio rural. La primera se caracteriza por el poco peso del área rural en el diagnóstico y regulación -POT 1999-. La segunda, por la adaptación y ajuste del POT (2006), etapa que propicia elementos de diagnóstico, regulación y presencia de las zonas rurales en relación con las zonas urbanas en el ordenamiento territorial. Y, la tercera, consistente en una variedad de instrumentos de planeación territorial con alta influencia en las zonas rurales del municipio. Esto ha dado lugar a una serie de inquietudes entre los actores sociales que interactúan en el territorio de estudio, que han quedado 
reflejadas en el trabajo de campo. Estos momentos y sus resultados se logran identificar en los aportes de los documentos consultados para esta investigación, así como en algunas de las entrevistas a expertos.

La política de Medellín sobre los corregimientos se ha debatido en concebirlos como un espacio en proceso de urbanización o concebirlos como un espacio de producción agraria. Ahí está dividida la política, por eso ha sido tan errática. Por otro lado, los procesos han sometido a un desgaste tremendo a las organizaciones y los líderes. Les podría comentar al menos cinco procesos de planificación tremendamente demandantes para las comunidades que se están llevando simultáneamente o que se llevaron en los últimos años. Es el caso de los Planes Especiales de Ordenamiento Corregimental, los Planes de Desarrollo Rural Sostenible o la Estrategia de Hábitat Rural Sostenible.

Así, se pasa de una etapa donde la política de planeación rural municipal se centraba en Medellín, y que consideraba el área rural como zona de expansión, a una etapa caracterizada por la cantidad y multiplicidad de instrumentos de la planeación para los corregimientos, con efectos de diferente índole cada uno. De esta forma, es muy reciente en el contexto de la ciudad de Medellín y sus cinco corregimientos la aplicación de una serie de políticas e instrumentos para la planeación de las áreas rurales desde la participación y vinculación de sus habitantes -por sus escasos años de formulación y aplicación-.

\section{Marco teórico y metodológico}

Para comprender el alcance de los resultados de la investigación es imprescindible llevar a cabo previamente una aproximación a algunos de los conceptos básicos que han servido de sustento para su formulación: la justicia ambiental y espacial, la noción de ruralidad y la comunicación para el cambio social. Asimismo, se presenta de manera sucinta la metodología utilizada que, en este caso, se ha considerado algo más que una guía para la recolección de información y el análisis.

\subsection{Justicia ambiental y espacial, entre la identidad y el reconocimiento}

El primer concepto con el que se ha trabajado, por considerarlo directamente ligado al ordenamiento territorial y a la cuestión de lo rural en este, ha sido el de justicia ambiental. La mayoría de autores consideran esta corriente como un ecologismo que, más allá de la reverencia de la naturaleza, pretende promover la conservación del medio ambiente por un interés material, considerándolo fuente y condición para el sustento de la comunidad (Martínez Alier, 2011, p. 34).

Aunque, de por sí lo anterior ya sería suficiente razón para el uso del concepto de justicia ambiental, en el marco teórico de un proyecto que trabajará en un escenario de ruralidad (o ruralidad metropolitana) amenazada, existen definiciones más completas que permiten relacionar esta idea con los otros dos pilares de la investigación: la comunicación para el cambio social y las metodologías de investigación social participativas.

En este caso, se ha tomado la definición de justicia ambiental que considera que esta va más allá de la búsqueda de una distribución apropiada de los costes y beneficios 
ambientales, al incluir elementos como el reconocimiento asociado a la identidad colectiva y la participación entendida como capacidad de influencia en la toma de decisiones:

Los movimientos demuestran que toda noción de justicia ambiental debe centrarse no sólo en las injusticias distributivas, sino en los aspectos de reconocimiento, participación y capacidades. El amplio alcance de la justicia ambiental demuestra la posibilidad de una no menos amplia e integrada noción de justicia; un proyecto político comprehensivo que incluya cuestiones de distribución, reconocimiento cultural, participación política, y también de capacidades y funcionamiento (Schlosberg, 2011, p. 34).

Este concepto se afianzó en el marco teórico asociado a la defensa de una ruralidad que ha resultado ser el principal elemento del reconocimiento y la identidad común de los habitantes de los corregimientos de Medellín. El mismo Martínez Alier relaciona algunos movimientos de justicia ambiental con el resultado lógico del desplazamiento geográfico de los impactos ambientales desde el centro de las ciudades a las periferias:

No existe una tendencia interna espontánea hacia el uso de formas sustentables de energía, o hacia una menor producción de residuos materiales, porque las protestas internas contra las externalidades en las ciudades muchas veces han sido desplazadas a otros lugares por los cambios en la escala (Martínez Alier, 2011, p. 212).

No obstante, queda por exponer un término importante que sitúa la comunicación, en su acepción más amplia, como un aspecto central de los conflictos de carácter ambiental. Martínez Alier (2011) hace énfasis en la importancia del lenguaje en este tipo de escenarios a través de lo que ha denominado "lenguajes de valoración". El autor pone en duda la reducción a la dimensión monetaria de las diferentes alternativas en los grandes proyectos, muchas veces asociados al ordenamiento territorial, y reivindica la importancia de incluir en la toma de decisiones "el pluralismo de valores inconmensurables" (p. 99):

Debemos rechazar tal simplificación de la complejidad, tal exclusión de lenguajes de valoración. Debemos aceptar, por el contrario, el pluralismo de valores inconmensurables entre sí para evitar que la ciencia económica se convierta en un instrumento del poder en la toma de decisiones (Martínez Alier, 2011, p. 99).

En resumen, la noción justicia ambiental está directamente relacionada con la identidad colectiva como reconocimiento, y con la capacidad de tomar decisiones por parte de las comunidades, que se vería facilitada si los lenguajes de valoración fueran más allá de la mera dimensión monetaria.

Tal y como se utiliza en esta investigación, el concepto de justicia ambiental no puede disociarse del concepto de justicia espacial, que incorpora las consideraciones de justicia social a los métodos geográficos de análisis (Harvey, 1977, p. 97). Este permite aproximarse a fenómenos como los períodos de gran crecimiento de las ciudades, directamente relacionados con el papel que desempeña la urbanización en la absorción de excedentes de capital, dificultando el acceso de grandes bolsas de población a unas mínimas condiciones de vida. 
Es a través del cambio de perspectiva que permite la justicia espacial que empieza a exigirse, más allá de un mayor control del excedente por parte del Estado, un control democrático del urbanismo para evitar que la configuración de las urbes quede en manos de élites que configuren el espacio en función de sus propias necesidades. Este fenómeno de expansión y "destrucción creativa" está directamente relacionado con lo que sucede en las periferias rurales de las grandes ciudades. Como ya adelantara Lefebvre, la fuerte expansión de las ciudades desplaza a la población de los alrededores, que se ve desposeída a causa de la industrialización y la comercialización de la agricultura (Lefebvre, 2013), mientras se difumina la distinción entre campo y ciudad dando lugar a espacios "porosos con un desarrollo geográfico desigual bajo el dominio del capital y del Estado" (Harvey, 2013, p. 32-45).

En conclusión, aunque el espacio en sí mismo esté básicamente dado, su organización y su significado son productos de la experiencia, la transformación y la dinámica social (Soja, 2010, p. 85 -87). Dicho de otro modo, el espacio y la organización política del espacio expresan las relaciones sociales pero también influyen en ellas ${ }^{2}$. Así, Scholberg desde la justicia ambiental y, Lefebvre, Harvey y Soja, como autores base del derecho a la ciudad y de la justicia espacial, nos presentan una ruta analítica para abordar los cambios socioespaciales que acontecen en las ciudades contemporáneas y dentro de estas, y en forma particular para este artículo, en las zonas rurales de Medellín.

\subsection{Sobre el concepto ruralidad en este artículo}

Precisamente ese carácter de proceso inacabado y en continua construcción enriquece el debate sobre las mismas características constitutivas de la ruralidad en un mundo globalizado en el que las reglas de la economía cambian de manera vertiginosa. Desde las perspectivas geográfica y sociológica continuamente se está replanteando qué deben cumplir un paisaje y sus comunidades para seguir considerándose rurales o si el mismo término ruralidad sigue manteniendo su vigencia. A esto hay que enfrentar, a su vez, las diferentes maneras en que las administraciones y los propios habitantes de las zonas más alejadas de las ciudades delimitan y consideran los espacios sobre los que interactúan.

Esta complejidad se incrementa exponencialmente en los bordes de las grandes ciudades, en los que interactúan y confluyen actores sociales que traen consigo diferentes prácticas sociales, formas de subsistencia y modos de vida tan diversos como sus características socioeconómicas y culturales. En espacios en los que se combinan características de lo urbano y lo rural con una importante riqueza ambiental, estos se disputan los usos del territorio y comparten y compiten hasta por la misma identidad, como veremos más adelante.

Autoras como Edelmira Pérez (2001, 2004) en Colombia, Blanca Ramírez (2003) en México y Claudia Barros (1999) en Argentina han indagado sobre las interacciones y cambios que se dan en las zonas rurales en contextos de metropolización (Egio y Torrejón, 2014). Barros, a partir del estudio de la periferia de Buenos Aires, reivindica el concepto de espacio rururbano como una forma de matizar la dureza de la oposición conceptual 
urbano-rural en territorios donde se intercalen diversas formas de organización territorial vinculadas con ambas formas de vida $(2000,2006)$. Por su parte, Pérez indaga en una ruralidad que "trasciende lo agropecuario", que no deja de serlo por mantener una fuerte relación con la ciudad sino que se asume que esta relación va más allá del intercambio de alimento, estando unida a la provisión de bienes y servicios (2001, 18).

La investigadora colombiana analiza la evolución del término rural desde el siglo XVIII, fuertemente asociado a la idea de progreso de la economía clásica, que asume que la transformación lógica estructural es de lo rural a lo urbano y de lo agrícola a lo industrial, de manera que se opone lo atrasado (rural) a lo moderno (urbano).

Frente al paisaje rururbano, Agudelo habla en el caso de Medellín de una ruralidad metropolitana caracterizada por espacios urbanizados en los que abundan actividades agroindustriales, industrias rurales, segundas residencias, comercios y grandes infraestructuras, pero que mantienen una matriz "esencialmente verde" e importantes elementos agrarios y que se relacionan con la ruralidad tradicional. Es decir, se trata de un paisaje que no termina de encajar con los rasgos del paisaje urbano. La ruralidad quedaría determinada por un cierto mantenimiento del paisaje más verde, aunque el verde lo aporte el césped de las casas de recreo, pero también por una cierta hibridación entre las costumbres de los habitantes originarios del campo y los de la ciudad (Agudelo, 2012).

La ruralidad, lejos de ser una realidad estática y definida, es una construcción social. Por lo tanto, se ha trabajado en esta investigación con un objeto de estudio cambiante que deberá ser analizado como tal, puesto que la ruralidad, como resultado de acciones y presiones colectivas, se construye y reconstruye contantemente, no deja de inventarse y debe ser analizada desde perspectivas que tengan esto en cuenta (Entrena 1998, 13-14).

\subsection{Comunicación para el cambio social, la visibilización de los discursos}

Como en todo proceso social complejo, en la expansión urbana de Medellín intervienen un gran número de actores sociales con intereses colectivos y particulares, en este caso relacionados con las políticas de ordenamiento territorial. La posición y el discurso de cada uno al momento de habitar, participar y decidir sobre su territorio están directamente relacionados con las formas en que son percibidos los efectos sociales, culturales, ambientales y económicos de dichas políticas.

Es por esto que la mera visibilización de los discursos y las posiciones puede ser un primer paso para la resolución de conflictos relacionados con la planeación territorial. En este sentido, resultan útiles los aportes de la comunicación para el cambio social, cuyos principios básicos, según varios autores, están directamente relacionados con la emergencia de las diferentes voces sobre un tema: (i) se centra en el proceso, (ii) privilegia el autorreconocimiento y empoderamiento de las comunidades y (iii) es una herramienta para la voz y la posición de los actores (Rodríguez, 2002; Gumucio, 2004).

Es decir, la comunicación para el cambio social se enfrenta al reto de ser "un vehículo para catalizar procesos de cambio, empoderar individuos, fortalecer comunidades y 
liberar voces que no han sido escuchadas previamente" (Rodríguez, 2002, p. 25). Pretende, por tanto, convertir a la misma comunidad en agente de su propio cambio, evitando que los expertos técnicos, ajenos al territorio, sean quienes guíen los procesos y finalmente tomen las decisiones por ellos.

En este sentido, la comunicación participativa (como elemento central de la comunicación para el cambio social), unida al uso de metodologías de investigación social (asimismo participativas), resulta de particular interés en el territorio de estudio:

Especialmente en comunidades que han sido marginadas, reprimidas o simplemente postergadas durante décadas, la comunicación participativa contribuye a infundir autoestima y orgullo por la cultura. Refuerza el tejido social a través del fortalecimiento de las organizaciones propias a la comunidad. Protege la tradición y los valores culturales, al mismo tiempo que facilita la integración de nuevos elementos (Gumucio, 2001, p. 37).

El desarrollo de procesos participativos en los corregimientos de Medellín constituye en sí mismo un elemento que facilita la apropiación por parte de la comunidad de temas que les conciernen y en los que pueden ejercer una gran influencia; así, busca generar acciones que contribuyan a mejorar la calidad de vida de las personas e "impulsarlas a negociar el avance a través de procesos, asociaciones y alianzas" (Rodríguez, 2002, p. 25).

[...] la comunicación para el cambio social no pretende definir anticipadamente ni los medios, ni los mensajes, ni las técnicas, porque considera que es del proceso mismo, inserto en el universo comunitario, del que deben surgir las propuestas de acción (Gumucio, 2011, p. 33).

En la investigación, el enfoque descrito permitió reconocer las diferentes dinámicas de la expansión urbana a través de procesos participativos focalizados "sobre las personas, sobre las comunidades y sobre las relaciones equilibradas y justas que deben establecerse para lograr transformaciones necesarias que permitan alcanzar metas concertadas de vida", donde el protagonismo principal no está centrado en "el modelo [...] pues este pierde relevancia y lo que importa es la forma como la sociedad se teje para buscar su futuro, y en ello la comunicación es la costura, la dinámica y la fuerza" (Cadavid, 2011, p. 77).

El uso de la comunicación para el cambio social como plataforma teórica para explorar las dinámicas de la expansión urbana en Medellín, se vinculó en forma directa con (i) la manera en que es percibido el proceso de ordenamiento territorial, (ii) el autorreconocimiento, y (iii) la voz y participación de los actores sociales que habitan o influyen en los corregimientos. Sirvió este enfoque de la comunicación para potenciar las técnicas de investigación participativa implementadas, descritas en el siguiente apartado, y como fondo, no solo la indagación en un asunto que definirá la forma de vida de los habitantes del territorio, sino el aporte de un camino más para el cambio y la transformación social.

\subsection{La investigación social participativa como metodología}

Partiendo de la idea de que las diferencias en la distribución de los beneficios y perjuicios ambientales tienen parte de su origen en procesos sociales, culturales, económicos y po- 
líticos, se hace imprescindible entonces para el análisis el uso de metodologías y técnicas tanto de las ciencias sociales como del cercano campo de la comunicación que permitan la identificación de las estructuras, las prácticas, las reglas, las normas, el lenguaje y los símbolos que actúan como mediadores de las relaciones sociales (Schlosberg, 2011, p. 27).

En este caso, se escogió con este fin la metodología de investigación social participativa desarrollada en Madrid por el Observatorio Internacional de Ciudadanía y Medio Ambiente Sostenible (CIMAS). Entre sus principios está el considerar que, en los conflictos ambientales, es importante el conocimiento técnico pero también lo son los valores, normas y expectativas de la población; por lo que más allá de abarcarse desde una dimensión técnica y científica, en la que los expertos jueguen un papel fundamental, estos deben tratarse también desde una dimensión "valorativa de carácter social y cultural" (Martín y Garrido, 2006, p. 256).

Si nos centramos en una definición amplia de la comunicación, como comunicación interpersonal, se puede deducir que el trabajo de investigación participativo y comunitario puede ser una valiosa herramienta pedagógica que facilite la reflexión ciudadana sobre problemas de cariz ambiental tan complejos como la construcción de la ciudad y el territorio. Es decir, que el propio proceso investigativo genera en sí mismo una acción transformadora, en este caso sobre su objeto de estudio que es la propia sociedad.

Por otro lado, para terminar de relacionar la metodología escogida con el marco teórico, no puede olvidarse que las herramientas participativas de investigación tienen un claro carácter comunicativo, coincidente con el modelo participativo de la comunicación: "El propósito [del modelo de comunicación citado] es la emancipación de las personas para que tengan un mayor control sobre las decisiones que les afectan y de esta manera potenciar la equidad y las prácticas democráticas" (De Bustos, 2006, p. 159).

\section{Sociogramas: posiciones y actores sociales}

La base de este artículo es el análisis de veintidós entrevistas semiestructuradas llevadas a cabo a diferentes actores sociales que habitan e influyen sobre la planificación territorial de los cinco corregimientos del municipio de Medellín. Para comprender bien cómo se escogieron estos actores es imprescindible reconocer cómo se elaboraron los mapas sociales (o sociogramas) en los que fueron identificados, entendiendo estos últimos como representaciones gráficas de las relaciones que existen entre grupos, instituciones o personas (Villasante, 2006, p. 415). Estos fueron construidos colectivamente por personas vinculadas a organizaciones sociales de los cinco corregimientos de Medellín, elegidas previamente según una serie de perfiles que se consideraron representativos tras la consulta de bibliografía y expertos. En total, se realizaron cinco talleres de elaboración de mapas sociales, uno por corregimiento.

A diferencia de otros análisis de redes, estos sociogramas se diseñaron participativamente respondiendo a cuatro variables. La primera fue la manera en que se situó el tema de estudio, que ocupó un espacio preferente en lugar de hacerlo un sujeto o actor central. A partir de aquí, los actores sociales identificados se situaron según la posición 
ideológica, estratégica o programática adoptada respecto a este. En este caso, las posturas respondían a un gradiente entre dos extremos, la defensa de un futuro rural o urbano del corregimiento, dejando ambos conceptos abiertos para que fueran los mismos participantes los que les aportaran un sentido. La segunda variable era de carácter relacional, centrada en los vínculos emotivos existentes entre los diferentes actores sociales identificados (Villasante, 2010, p. 50).

También se tuvo en cuenta, como otra variable, el poder simbólico u organizativo, previendo que unos actores dispondrían de una estructura formal organizativa (asociaciones); otros, como instituciones o corporaciones, además de organizados ostentarían un poder al menos simbólico y, al tiempo, una parte importante de la sociedad no estaría organizada. En este sentido se distinguió entre instituciones o imágenes de poder, sociedad organizada o tejido asociativo y sociedad no organizada o base social. La última de las variables tenía que ver con la capacidad de influencia de cada actor con respecto al tema estudiado. Esta se considera la más estructural porque depende de elementos históricos y heredados de situaciones previas, estando muchas veces relacionada con el poder económico o de clase social (Villasante y Martín, 2007, p. 130).

Fueron precisamente los propios sistemas de relaciones identificados en los sociogramas los que señalaron cómo había de ser la selección de los elementos de la muestra a entrevistar (Villasante y Martín, 2007, p. 132). En este caso se contó con miembros de órganos comunitarios (como Juntas Administradoras Locales), campesinos, adultos mayores, expertos en desarrollo local en Medellín, funcionarios de las administraciones locales, amas de casa, jóvenes de asociaciones culturales y miembros de medios comunitarios, entre otros.

\section{CORREGIMIENTO DE SAN SEBASTIÁN DE PALMITAS \\ Los actores sociales y el territorio}
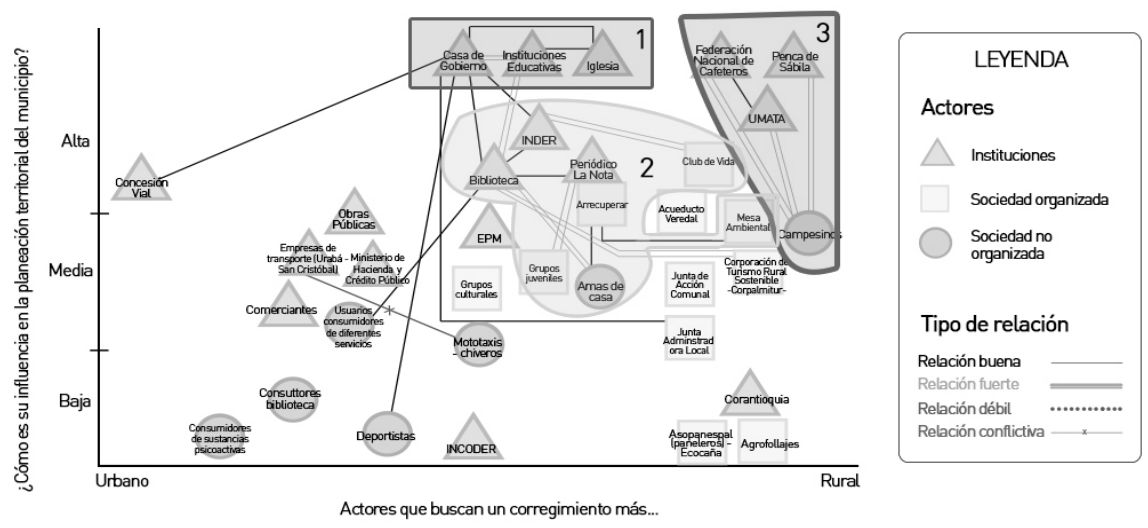

Gráfica 1. Sociograma diseñado en San Sebastián de Palmitas.

Fuente: laboración propia 
Las entrevistas se llevaron a cabo de manera individual en el espacio de cotidianidad de cada uno de los actores, aunque previamente algunos ya habían participado en los talleres de elaboración de mapas sociales. Siguiendo la filosofía de la investigación participativa, en una última etapa se llevaron a cabo tres talleres de devoluciones creativas en las que se presentaron los resultados de las entrevistas en forma de multilemas o juegos de frases anónimas para continuar con la indagación colectiva. No obstante, los resultados obtenidos de esta última etapa merecerían un artículo propio y no pueden desarrollarse en este texto.

\section{Resultados}

Para llevar a cabo un análisis ordenado del contenido surgido en las entrevistas, este se dividirá en tres de las características, ya descritas en el marco teórico, de los conceptos de justicia ambiental y espacial. De este modo se profundizará, a través de los testimonios de los entrevistados, en las percepciones sobre la identidad en los corregimientos (i), sobre la participación en el ordenamiento territorial (ii) y sobre la manera en que se considera que se distribuyen los costos y beneficios ambientales asociados al modelo de desarrollo (iii). Tratando de respetar los principios de la comunicación para el cambio social, se dará un especial protagonismo a las frases literales surgidas en las conversaciones.

Hay que recordar que, siguiendo los resultados de los sociogramas, entre los veintidós entrevistados hay personas que habitan los cinco corregimientos y actores que inciden en su ordenamiento territorial sin necesidad de habitar allí. Se procuró cumplir con la diversidad de perfiles de un espacio rural metropolitano en el que convive tanto población rural tradicional como urbana reciente.

\subsection{Identidad: el reconocimiento de la ruralidad y la actividad agropecuaria}

En un territorio tan amplio y diverso como lo son los corregimientos del municipio de Medellín no puede obviarse la presencia (o emergencia) de identidades múltiples ligadas a la complejidad del propio entramado social. En este sentido, antes de abordar este punto resulta interesante echar un vistazo somero a la reflexión al respecto que ha hecho la Geografía Regional, una disciplina que en este caso aporta una perspectiva complementaria que enriquece aún más el análisis del objeto de estudio. Al debate entre lo "urbano" y lo "rural", que podría considerarse central en un territorio híbrido, se unen nuevas variables en los actores sociales que habitan los corregimientos.

La lógica posmoderna ha propiciado que la cultura deje de ser vista como un conjunto relativamente uniforme y normativo de creencias, valores, actitudes, comportamientos y productos. Minorías y/o grupos subalternos cuyas voces habían sido anteriormente excluidas reclaman ahora atención como partes esenciales del sistema social: las variables de género, de clase, de etnia, de edad, de condición física, etc. (Albet, 2001, p. 46).

Precisamente por esa diversidad esperada resulta especialmente significativo que un eje vertebrador muy claro de la identidad en los corregimientos de Medellín, casi 
independientemente del grupo social que se trate, sea la importancia central que se les atribuye a la agricultura, la ruralidad y la vida campesina. Esta idea se expresa en el siguiente testimonio.

Yo hace doce años vivo acá en el corregimiento. Yo a Medellín no quiero volver: mucho cemento, mucha contaminación. Lo rural no lo tiene, la ruralidad tiene la ventaja de que si estás enfermo de los pulmones te alivias, si estas deprimido te alivias, porque el panorama cambia, el ambiente cambia (Hombre adulto. Habitante de Santa Elena).

A pesar de la diversidad de actividades económicas ejercidas en los corregimientos, cuando se pregunta a los actores sociales presentes en el territorio por los aportes al resto del municipio, se destaca la conservación de los servicios ambientales, la función como despensa agrícola e incluso el mantenimiento de una cultura alternativa. Quedan olvidados, por tanto, los usos industriales, comerciales, residenciales o para otros equipamientos que se mantienen en este espacio.

Las bondades más importantes del corregimiento son desde la parte ambiental donde se produce agua, oxígeno y temas de diversidad de especies y cultura. Un aspecto cultural también (Hombre adulto. Habitante de Santa Elena).

Entonces cada uno desde el punto de vista alimentario le está aportando una gran cantidad de alimentos. Podemos estar hablando de una muy buena participación en el mercado por parte de los pequeños, medianos y grandes productores (Hombre adulto. Habitante de San Antonio de Prado).

Aunque se trate de un espacio rural urbanizado, la ruralidad aparece como un factor diferenciador del resto del municipio, elemento por tanto nuclear de la identidad. Se trata de una identidad que se percibe en peligro y que muchas veces se expresa precisamente a partir del miedo a su desaparición. Viva donde viva el entrevistado, se dedique a lo que se dedique, se coincide en destacar que se trata de un bien a conservar.

Yo pienso que en ese tema Altavista tiene deficiencias porque la mayoría de hombres y mujeres tienen que desplazarse a la ciudad para poder buscar su sustento económico; o sea, laboralmente tienen que salir del corregimiento generando así la pérdida de identidad campesina (Mujer adulto mayor. Habitante de Altavista).

Ya el muchacho no es del campo. Ya está a 45 minutos de Medellín pero tiene también las presiones de estos dos valles. Entonces como esto se va conurbando y yo no soy ni urbano ni rural, entonces, ¿yo qué soy? (Hombre adulto. Habitante de Santa Elena).

Siguiendo esta misma lógica, se convierte en un escenario deseable un futuro en el que primen la agroecología, el ecoturismo y otras actividades que pudieran compatibilizar el desarrollo económico con el mantenimiento de la actividad agrícola.

Yo me soñaría un corregimiento que integre distintas actividades económicas de beneficio para la comunidad y que se pudiera conservar la actividad agrícola dándole un manejo ambientalmente sostenible. (Mujer adulta. Habitante de San Cristóbal). 
Entonces se puede explotar eso como agroturismo, como turismo ecológico, turismo ambiental, porque contamos con una reserva que es la Reserva del Romeral (Hombre joven. Habitante de San Antonio de Prado).

Paradójicamente, cuando se habla de este tipo de actividades económicas, la cercanía a la ciudad, que como se verá más adelante suele expresarse como una amenaza, se convierte al tiempo en una oportunidad como mercado.

Desde el tema turístico vemos un potencial hacia la ciudad de Medellín, hacia ella estamos trabajando. Porque la gente no sabe cómo se producen los alimentos. Se invita a que salga de esa ciudad tan estresante y venga a los alrededores, que la gente venga, disfrute de la naturaleza, de las fuentes hídricas (Hombre adulto. Habitante de San Cristóbal).

Ahondado en esta idea, el que la actividad agropecuaria sea una seña de identidad de los corregimientos, por muy urbanas que sean las dinámicas de algunas centralidades, hace que el campesino, como elemento clave en su mantenimiento, ocupe un lugar destacado para el resto de los habitantes. Todos los actores sociales que habitan los corregimientos coinciden en resaltar la crisis de la agricultura tradicional y solicitar que se mantenga el trabajo campesino mediante la ayuda de las administraciones públicas. De este modo, se trata de defender una identidad que se considera como propia pero que mantienen otros.

Los campesinos y las campesinas para poder estar ahí y seguir con esa tradición tendrían que tener generada unas garantías con condiciones aptas para vivir y no tener que irse para otro lado a buscar empleo (Mujer adulto mayor. Habitante de Altavista).

Es decir, el no campesino, en muchas ocasiones venido de la ciudad, mira al campesino como valedor de la identidad y expone como ideal su permanencia en el territorio. Sin embargo, es paradójico que quien trabaja directamente la agricultura muchas veces aspira a que sus hijos abandonen su actividad económica como paso imprescindible para conseguir una vida más próspera. La ciudad, cercana, es percibida así como fuente de servicios y oportunidades con los que normalmente no cuenta el mundo rural próximo.

Porque como yo en Medellín tengo familia que está viviendo allá. Es una cosa que yo agradezco, que estudiaron y se fueron para allá y consiguieron su trabajo (Hombre adulto mayor. Habitante de San Sebastián de Palmitas).

De igual forma piensan los hijos de campesinos.

Tener esa ciudad tan grande, una ciudad como Medellín, a cuarenta minutos de esto tan rural, eso es una gran ventaja, sin duda alguna; porque podemos acceder a la educación, a servicios, la gente puede ir a trabajar, volver al corregimiento (Mujer joven. Habitante de San Sebastián de Palmitas).

Relacionado con lo expuesto en este apartado, en el último taller de trabajo comunitario, llevado a cabo en el corregimiento de San Cristóbal con participantes de este corregimiento y de San Sebastián de Palmitas, entre todos los asistentes se llegó por consenso a la siguiente frase: 
Estamos construyendo un corregimiento que mantendrá la actividad agrícola, ambiental y sostenible para que los campesinos permanezcan en su territorio; a través de la articulación y la unión de campesinos y comunidad, generada por una propuesta educativa liberadora y transformadora.

De esta manera, quienes decidieron formar parte del taller resumieron algunas de las ideas aquí expuestas, entre ellas la necesidad de mantener la agricultura en los corregimientos, el que se haga mediante técnicas sostenibles ambientalmente y la necesidad de unir esfuerzos entre la comunidad campesina y quienes se dedican a otras actividades económicas. Es decir, en un espacio en el que conviven actividades agrícolas, pecuarias y de protección ecológica, pero también industriales, comerciales, residenciales y recreativas, los entrevistados prefirieron destacar las primeras, asociadas tradicionalmente a los territorios rurales.

Es decir, aunque en la actualidad los paisajes rurales no tienen por qué ser necesariamente agrarios, la agricultura sigue siendo un elemento fundamental para que sus habitantes consideren que el territorio sigue siendo rural, confluyendo los intereses de nuevos y antiguos residentes.

\subsection{Participación: muchos procesos dispersos en el territorio y pocos resultados}

El reconocimiento de la identidad está muy ligado a la capacidad de participación y decisión sobre las actuaciones que definirán los usos del suelo de una zona, puesto que este tendrá importantes repercusiones sociales, económicas y ambientales. En el caso estudiado, al tratar el ordenamiento territorial con los actores sociales que habitan los corregimientos adquiere en la conversación una especial relevancia la necesidad de que la visión local sobre el territorio sea tenida en cuenta por la administración. Es decir, quien habita los corregimientos pide que su opinión no solo sea escuchada, sino que tenga un impacto real sobre la planificación.

En este sentido, se admite que existen desde la administración local escenarios abiertos para la participación ciudadana; sin embargo, los entrevistados destacan que estos procesos no han dado como resultado la priorización de las propuestas surgidas desde las comunidades de la periferia.

Muchas veces lo que prioriza la comunidad no se tiene en cuenta porque priman las políticas de turno más que lo que prioriza la misma comunidad como tal (Hombre adulto. Habitante de Altavista).

Por otro lado, la unión entre participación, reconocimiento y capacidad de decisión se expresa también a través de la especial importancia que se le concede a la inclusión de los corregimientos en la cartografía del municipio.

Antes no aparecían, o sea, hace dos o tres años no aparecíamos en el mapa y apenas el año pasado se está hablando de campesinos que viven en la ciudad de Medellín (Hombre adulto. Habitante de Santa Elena). 
Pero la crisis de reconocimiento va más allá de las administraciones públicas locales y afecta también a los propios órganos de representación comunitaria. Algunas personas destacaron que, aunque se han conseguido avances, es difícil la inclusión en estos de algunas minorías como afrodescendientes, jóvenes, mujeres o discapacitados.

Entonces, hasta hace poquito estaba hablando con uno de esos señores y me dijo que no, que de parte de juventudes que solamente hubiera un representante. Y yo es que no, entonces, ¿̇ustedes de la tercera edad cuántos cupos tienen? (Hombre joven. Habitante de San Antonio de Prado).

Deben ser procesos incluyentes, donde todos y todas estén ahí inmersos, incluidos e incluidas, porque no tiene razón de ser que sea la voz de unos cuantos y cuantas (Mujer adulto mayor. Habitante de Altavista).

Asimismo, se reconoce cierta tendencia a la dispersión de un tejido asociativo y comunitario muy diverso, incluso cuando los fines perseguidos puedan ser en algunos casos muy similares. De hecho, en los encuentros se resaltó cómo los intereses particulares contribuyen a generar contradicciones.

Hay una gran mayoría que está con el tema con lo rural pero en su mayoría no hay relaciones fuertes. Hay incluso relaciones de conflicto, como es el caso de la Junta Administradora Local que a pesar de tener un papel importante está desarticulada con las otras organizaciones (Trabajadora de una entidad sin ánimo de lucro. Santa Elena).

Esto provoca una falta de comunicación entre los actores que dicen tratar de mantener la naturaleza rural de los corregimientos. Incluso en algunos casos el conflicto fue palpable durante el propio ejercicio.

No hablo porque hay aquí un representante de esa asociación (Hombre adulto. Habitante de San Antonio de Prado).

Al igual que sucedía en relación con la identidad, en uno de los encuentros finales, con asistentes de los corregimientos de Altavista y San Antonio de Prado, la frase de síntesis elaborada por los asistentes coincidía con lo expresado en las entrevistas.

La gente se va cansando de tantos procesos y se desliga del poder, y otro se apropia de las decisiones.

Se destacaba, por tanto, que son muchos los procesos participativos abiertos desde las administraciones públicas y que la falta de resultados claros provoca que los ciudadanos se abstengan de participar, perdiendo definitivamente su capacidad de decisión sobre la planificación territorial. Esto coincide con lo expresado por algunos teóricos de la comunicación para el cambio social: en estos procesos la comunicación "ha sido marginada de los programas de desarrollo la mayor parte de las veces, y cuando no ha sido el caso, se ha convertido en un soporte institucional o en un instrumento de propaganda" (Gumucio, 2004, p. 4). 


\subsection{Distribución de costes y beneficios ambientales: la ciudad como amenaza}

Finalmente las percepciones surgidas en los dos puntos anteriores son el resultado de un modelo de desarrollo cuya distribución de costes y beneficios (ambientales pero también económicos) es percibida como inequitativa. Los participantes (y algunos funcionarios entrevistados) coincidieron en afirmar que mientras que los corregimientos tienen un importante papel en el aporte de servicios ambientales a la ciudad, asumen, por el contrario, una elevada carga en relación con los usos del suelo para fines residenciales e infraestructuras.

Este análisis se puede unir al de la literatura especializada, según la cual los diferentes instrumentos de planificación y ordenamiento territorial han supuesto un aumento del suelo de expansión. Este habría conllevado la implantación de usos urbanos que habría vuelto más atractivo a los ojos de los citadinos este territorio que empezaría a combinar calidades ambientales de la vida rural con comodidades de la vida urbana (Agudelo, 2012, p. 561), contribuyendo así a aumentar la densidad de población.

$\mathrm{Al}$ respecto, los habitantes tradicionales consideran que los corregimientos son olvidados por la administración al autorizar nuevas construcciones pero no mejorar los servicios públicos.

Esos seiscientos apartamentos conllevan veinticuatro mil personas más en población, porque mínimo vienen papá, mamá y dos muchachos. Entonces carecemos de espacios de educación, carecemos de espacios de cultura-educación, de esparcimiento, entonces es un problema social (Mujer adulta. Habitante de San Antonio de Prado).

Por otro lado, suelen exigirse contraprestaciones por la conservación de espacios naturales que ofrecen servicios ambientales a la ciudad. Es posible que esta petición tenga que ver con la influencia de años de trabajo con funcionarios del área ambiental en muchos de los proyectos de planificación participativa citados anteriormente.

Yo pienso que al mantener esa carga hídrica, forestal y como pulmón verde y como bien ambiental, deberíamos recibir retribución, que las personas pudieran estar en sus fincas y no tributar, sino recibir tributo, beneficio (Mujer adulto mayor. Habitante de Altavista).

En este último punto la frase elaborada conjuntamente para sintetizar la percepción común de los participantes fue la siguiente: "La ruralidad, un derecho para todos y no un beneficio para unos pocos".

\section{Conclusiones}

A pesar de la falta de coordinación entre organizaciones y de que no existiera en el momento de la investigación un movimiento activo y general de resistencia frente al modelo de desarrollo, las entrevistas llevadas a cabo a habitantes y actores que influyen sobre la planeación territorial en los corregimientos sacaron a la luz que existe un malestar extendido frente a este. En una misma postura coinciden miembros de movimientos ambientalistas, medios comunitarios, organizaciones de género, ONG, movimientos 
campesinos y organizaciones agrarias, e incluso miembros del funcionariado. Es decir, se da, o al menos se percibe, una defensa de las propiedades rurales del entorno incluso desde aquellos actores que no viven la ruralidad de una manera clásica.

Aunque se expresen de una manera difusa, las principales percepciones compartidas coinciden con los tres principios fundamentales del concepto de justicia ambiental, tal y como lo describe David Schlosberg. La preocupación por la pérdida de identidad, en este caso muy ligada a un problema ambiental; la reivindicación de reconocimiento y capacidad de participación real en la planificación del territorio y la denuncia de una distribución inequitativa de costos y beneficios ambientales fueron una constante entre los entrevistados. Coincide este resultado, en el entorno de una ciudad en expansión como Medellín, con esta frase acuñada por Luis Carlos Agudelo (2012): "El sueño de la vida en el campo se ha convertido en la pesadilla del suburbio" (p. 561). Es decir, la incontrolada expansión urbana en Medellín altera el derecho a la ciudad, en palabras de Lefebvre, dando lugar a un desarrollo geográfico desigual entre las zonas rurales y las zonas urbanas de la ciudad.

Por otro lado, acorde con las entrevistas y talleres realizados para esta investigación, surgen las identidades en los corregimientos como base de una cultura local que moldea el territorio a la luz de las tensiones y presiones de la expansión urbana de la ciudad de Medellín. Es decir, el peso y valor que se le da a la agricultura, bajo la figura del campesino y el escenario de la vida campesina, se han venido matizando y transformando en la medida en que la economía campesina ha disminuido cada vez más y se han hecho necesarias otras actividades. Esta pluriactividad -en referencia a la incursión de actividades de servicios en forma paralela a las actividades agrícolas- ha traído consigo a su vez una serie de cambios en la vida cotidiana de las familias en los corregimientos. La renta del suelo urbano es quizá, según los aportes de la teoría marxista, el principal motor de cambio para este proceso.

De esta manera, las culturas locales en términos de la geografía regional tienen una doble función social: una como base de cohesión a partir de elementos comunes de las identidades (identidad del lugar) y la otra como elemento de diferencia e hibridación con las otras culturas e identidades locales (sentido global de los otros lugares). Así, nuevamente las culturas locales, en este caso de cada uno de los corregimientos de Medellín, son particulares y significativas por su función de adaptación, transformación y/o resistencia frente al modelo de desarrollo urbano. Por ejemplo, y desde las grandes, poderosas y estandarizadas imágenes que se promocionan de la ciudad de Medellín, los corregimientos son vida campesina, flores, recursos naturales y patrimonio cultural.

Por otro lado, en este caso, tanto la comunicación para el cambio social como las metodologías de investigación participativa demostraron ser herramientas adecuadas de indagación en la percepción y las identidades de los actores sociales identificados. Muy cercanas entre sí, y basadas ambas en la comunicación interpersonal, sirvieron tanto para aflorar "sentires" como para reflexionar en conjunto sobre estos. 


\section{Bibliografía}

Albet, A. (2001). ¿Regiones singulares y regiones sin lugares? Reconsiderando el estudio de lo regional y lo local en el contexto de la geografía postmoderna. Boletín de la Asociación de Geógrafos Españoles, (32), pp. 35-52.

Agudelo, L. C. (2002). Indicadores de sostenibilidad y ordenación del territorio: Huella Ecológica y ecosistemas estratégicos en Medellín, Colombia. Recuperada el 13 de febrero de 2014. Disponible en

http://www.docentes.unal.edu.co/lcagudel/docs/INDICADORES\%20DE\%20SOSTENIBILIDAD\%20Y\%20 ORDENACION\%20DEL\%20TERRITORIO..pdf

Agudelo, L. C. (2012). Ruralidad metropolitana. Entre la tradición y el "brillo" urbano. Una interpretación. Bulletin de l'Institut Français d'Études Andines, 41, (3), pp. 555-571.

Barros, C. (2000). Reflexiones sobre la relación entre lugar y comunidad. Documents d'Anàlisi Geogràfica, (37).

Barros, C. (1999). De rural a rururbano: Transformaciones territoriales y construcción de lugares al Sudoeste del Área Metropolitana de Buenos Aires. Scripta Nova. Revista Electrónica de Geografía y Ciencias Sociales, 51, (45).

Barros, C. (2006). La ciudad en el campo: nuevas ruralidades y lugares rururbanos. In J. R. Nogué, J. (Ed.), Las otras geografías. Valencia: Tirant Lo Blanch.

Cadavid, A. (2011). La comunicación para el desarrollo en Colombia, los últimos 20 años. En G. Pereira; A. Cadavid (Ed.). Comunicación, desarrollo y cambio social. Interrelaciones entre comunicación, movimientos ciudadanos y medios. Bogotá: Pontificia Universidad Javeriana.

Cano, M. (2012). Percepción sobre las dinámicas rural-urbanas en el corregimiento de San Sebastián de Palmitas. Medellín: Universidad Nacional de Colombia.

Cruz Paz, M. S. (2003, enero-abril). Presentación. Sociológica, 18, (51), pp. 5 10.

De Bustos, M. (2006). Comunicación sostenible y desarrollo humano en la sociedad de la información. Consideraciones y propuestas. Madrid: Agencia Española de Cooperación Internacional.

Departamento Administrativo de Planeación (2006). Plan de Ordenamiento Territorial de Medellín. Acuerdo 46 de 2006. Medellín: Alcaldía de Medellín.

Departamento Administrativo de Planeación (2012). Plan de Desarrollo 2012 2015. Medellín un hogar para la vida. Medellín: Alcaldía de Medellín.

Departamento Administrativo Nacional de Estadística (2005). Censo general 2005. Nivel nacional. Recuperada el 13 de febrero de 2014. Disponible en

http://www.dane.gov.co/dane/censo/files/libroCenso2005nacional.pdf

Departamento de Geología Universidad de EAFIT (2010). El impacto humano. En: M. Hermelin; A. Echeverri; J. Giraldo (Eds.) Medellín: Medio Ambiente, Urbanismo y Sociedad. Medellín: Fondo Editorial Universidad EAFIT.

Egio, C. y Torrejón, E. (2014). Actores sociales y ordenamiento territorial rural. Bitácora Urbano/ Territorial, 2 (24).

Entrena, F. (1998). Cambios en la construcción social de lo rural. De la autarquía a la globalización. Papers. (56). 
Farah, M. A. y Pérez, E. (2004). Mujeres rurales y nueva ruralidad en Colombia. Cuadernos de desarrollo rural (51), pp. 138-160.

Gumucio, A. (2001). Haciendo olas: historias de comunicación participativa para el cambio social. Nueva York: The Rockefeller Foundation.

Gumucio, A. (2004, agosto). El cuarto mosquetero: la comunicación para el cambio social. Investigación y desarrollo, (12), pp. 2-23.

Gumucio, A. (2011). Comunicación para el cambio social: clave del desarrollo participativo. En: G. Pereira; B. Cadavid (Ed.) Comunicación, desarrollo y cambio social. Interrelaciones entre comunicación, movimientos ciudadanos y medios. Bogotá: Pontificia Universidad Javeriana.

Harvey, D. (1977). Urbanismo y desigualdad social. Madrid: Siglo XXI.

Harvey, D. (2013). Ciudades rebeldes. Del derecho de la ciudad a la revolución urbana. Madrid: Akal.

Lefebvre, H. (2013). La producción social del espacio. Madrid: Capitán Swing.

Llambí, L y Pérez, E. (2007, junio-diciembre). Nuevas ruralidades y viejos campesinismos. Agenda para una nueva sociología rural en América Latina. Cuadernos de desarrollo rural (59), pp. 37-61.

Martín, P.; Garrido, F.J. (2006). Metodologías participativas de investigación y planificación del medio ambiente. En L. Camarero (Ed.) Medio Ambiente y Sociedad. Elementos de explicación sociológica. Madrid: Thomson Editores.

Martínez Alier, J. (2011). El ecologismo de los pobres. Conflictos ambientales y lenguajes de valoración. Barcelona: Icaria Antrazyt.

Pérez, E. (2001). Hacia una nueva visión de lo rural. In CLACSO (Ed.), ¿Una nueva ruralidad en América Latina? Buenos Aires.

Pérez, E. (2004). El mundo rural latinoamericano y la nueva ruralidad. Revista Nómadas (20).

Pérez, H. (2011). Conflicto entre la declaratoria de áreas protegidas y las áreas rurales dedicadas a la producción agropecuaria -caso corregimientos de San Cristóbal y San Sebastián de Palmitas en el municipio de Medellín-. Recuperada el 13 de febrero de 2014. Disponible en http://tesis. udea.edu.co/dspace/handle/10495/1620

Piedrahita, C.; González, J.A. (2010). El ordenamiento territorial como mecanismo de transformación social: una mirada jurídica y política de la transformación urbana de la ciudad de Medellín. En M. Hermelin; A. Echeverri; J. Giraldo (Eds.) Medellín: Medio Ambiente, Urbanismo y Sociedad. Medellín: Fondo Editorial Universidad EAFIT.

Pradilla Cabos, Emilio (2002, abril-junio). Campo y ciudad en el capitalismo actual. Ciudades (54), pp. 3-8.

Ramírez, B. (2003, enero-abril). La vieja agricultura y la nueva ruralidad: enfoques y categorías del urbanismo y la sociología rural. Sociológica, 18, (51), pp. 49-71.

Rodríguez, C.; Obregón, R.; Vega, J. (2002). Estrategias de comunicación para el cambio social. Quito: Fundación Friedrich-Ebert-Stiftung Proyecto Latinoamericano de Comunicación.

Schlosberg, D. (2011). Justicia ambiental y climática: de la equidad al funcionamiento comunitario. Ecología Política. Cuadernos de Debate Internacional, (41), pp. 25-35.

Soja, E. W. (2008). Posmetrópolis. Estudios críticos sobre las ciudades y las regiones. Madrid: Traficantes de Sueños. 
Soja, E. W. (2010). La dialéctica socio-espacial. In N. Benach \& A. Albet i Mas (Eds.), Edward W. Soja. La perspectiva postmoderna de un geógrafo radical. Barcelona: Icaria.

Villasante, T. R. (2006). Desbordes creativos. Estilos y estrategias para la transformación social. Madrid: Catarata.

Villasante, T. R. y Martín, P. (2007). Redes y conjuntos de acción: para aplicaciones estratégicas en los tiempos de la complejidad social. Política y Sociedad, 1, (44), pp. 125-140.

Villasante, T. R. (2010). Conjuntos de acción y participación rural. Documentación Social, (155), pp. 43-58.

\section{Notas:}

1 División administrativa que comprende un área considerada rural cuya jurisdicción está supeditada a un municipio.

2 Edward Soja retoma y profundiza en las reflexiones de Lefebvre y hace énfasis en el papel del tiempo y lo histórico como un elemento fundamental de la trialéctica espacial, reivindicando el papel complejo y simultáneo que tienen las dimensiones social, histórica y espacial en la construcción de ciudad (Soja, 2008, p. 35). De esa relación trialéctica espacialidad-socialidad-historicidad es de donde surge lo que Soja denomina el Tercer Espacio.

3 Nos referimos a población rural dispersa, según el DANE, aquella que se da en un área caracterizada por la disposición dispersa de viviendas y explotaciones y que no cuenta, por tanto, con un trazado o nomenclatura de calles, carreteras, avenidas y demás.

$4 \quad$ Una presentación detallada del proceso metropolitano y los cambios de los perfiles poblacionales para algunas ciudad en América Latina son presentados en los aportes de Cruz (2003), Ramírez (2003), Barros (2006), Farah y Pérez (2004), Llambí y Pérez (2007), Pradilla (2009) y Agudelo (2012).

5 Entrevista a Luis Carlos Agudelo (marzo de 2013). Ingeniero forestal, Universidad Nacional de Colombia. Especialista en Ordenación del Territorio y Medio Ambiente. Doctor en Urbanismo, Territorio y Sostenibilidad. 